\title{
Dutasteride: an evidence-based review of its clinical impact in the treatment of benign prostatic hyperplasia
}

\author{
Andrew Thomson
}

Core Medical Publishing, Knutsford, UK

\begin{abstract}
Introduction: Benign prostatic hyperplasia (BPH) is a common condition affecting older men. Bothersome symptoms can progress to serious complications such as acute urinary retention (AUR) requiring surgical intervention. Dutasteride, a dual 5-alfa-reductase (5AR) inhibitor (5ARI), is a recently introduced therapy for the treatment of $\mathrm{BPH}$.

Aims: The objective of this article is to review the evidence for the treatment of BPH with dutasteride.

Evidence review: Evidence from large clinical studies shows that men with an enlarged prostate achieve a measurable decrease in prostate volume by up to $26 \%$ after 4 years of treatment with dutasteride and urinary symptoms improve after 6 months of treatment. This is achieved by rapid suppression (through inhibition of 5AR) of the principal androgen (dihydrotestosterone or DHT) responsible for stimulating prostatic growth. Evidence suggests that dutasteride treatment results in a reduction in risk (rather than delay) of the most serious complications including episodes of AUR and the need for BPH-related surgery. Early symptom relief has been achieved with the combination of an alfa blocker and dutasteride. There is good evidence that dutasteride is well tolerated; side effects limited to sexual dysfunction (reduced libido, impotence, and gynecomastia) are more common compared with placebo but occur with a similar incidence to finasteride, another 5ARI. No pharmacoeconomic evidence from studies with dutasteride has so far been published.
\end{abstract}

Clinical value: In conclusion, dutasteride is a valuable treatment option in men with moderate to severe $\mathrm{BPH}$. Reductions in prostate volume lead to symptom relief and serious complications appear to be reduced.

Key words: dutasteride, evidence-based review, benign prostatic hyperplasia (BPH), 5-alfa-reductase inhibitor

Core evidence clinical impact summary for dutasteride in benign prostatic hyperplasia

\begin{tabular}{|c|c|c|}
\hline Outcome measure & Evidence & Implications \\
\hline \multicolumn{3}{|l|}{ Patient-oriented evidence } \\
\hline Detectable symptom relief & Substantial & $\begin{array}{l}\text { Effective treatment; delay in onset of action may be reduced by short-term combination with } \\
\text { an alfa blocker }\end{array}$ \\
\hline Avoidance of surgery & Clear & $\begin{array}{l}\text { Avoidance of one of the most costly complications associated with the progression of benign } \\
\text { prostatic hyperplasia }\end{array}$ \\
\hline Reducing the risk of acute urinary retention & Clear & $\begin{array}{l}\text { Avoidance of a serious acute complication of benign prostatic hyperplasia which requires } \\
\text { prompt medical intervention }\end{array}$ \\
\hline Tolerability & Substantial & $\begin{array}{l}\text { Adverse events are few and reversible. Although prostate-specific antigen levels are reduced, } \\
\text { the "doubling rule" can be used and dutasteride use does not appear to interfere with the } \\
\text { detection of prostate cancer }\end{array}$ \\
\hline \multicolumn{3}{|l|}{ Disease-oriented evidence } \\
\hline Prostate gland size reduction & Substantial & Fundamental outcome in treating the disease rather than just providing symptomatic relief \\
\hline Dihydrotestosterone suppression & Clear & Reduced levels of the principal androgen responsible for prostatic growth \\
\hline \multicolumn{3}{|l|}{ Economic evidence } \\
\hline Cost effectiveness & No evidence & Studies required \\
\hline
\end{tabular}




\section{Scope, aims, and objectives}

Benign prostatic hyperplasia (BPH) is a common condition in older men. It is characterized by the presence of troublesome lower urinary tract symptoms (LUTS) which may be due to a number of factors. This condition, which can significantly affect quality of life, is becoming more prevalent as the population ages. There are a number of strategies for the treatment of $\mathrm{BPH}$ including surgical interventions and drug therapy. However, all of these interventions have a variety of advantages and disadvantages which will influence decisions made by patients and clinicians for the management of the condition. Dutasteride, a dual 5-alfa-reductase (5AR) inhibitor (5ARI), was introduced in 2002 for the treatment of $\mathrm{BPH}$.

The objective of this review is to provide an evidence-based appraisal of dutasteride for the treatment and management of $\mathrm{BPH}$.

\section{Methods}

Medical literature databases were searched for appropriate articles relating to dutasteride for the treatment of BPH. A search of the following databases was conducted on February 15, 2005 using the search terms "dutasteride; benign prostatic hyperplasia OR BPH AND guidelines" published between January 1990 and January 2005 (inclusive):

- PubMed, http://www.ncbi.nlm.nih.gov/entrez/query.fcgi

- EMBASE, http://www.datastarweb.com

- BIOSIS, http://www.datastarweb.com

- Database of Abstracts of Reviews of Effectiveness (DARE), http://www.york.ac.uk/inst/crd/darehp.htm

- Cochrane Database of Systematic Reviews (CDSR), www.cochrane.org/index0.htm

- Clinical Evidence, http://www.clinicalevidence.com

- National Institute for Health and Clinical Excellence (NICE), www.nice.org.uk

- National Guideline Clearing House, www.guideline.gov

A total of 77 articles (including abstracts from meetings and full publications) was identified and any animal, in-vitro studies, and non-English articles were excluded.

Table 1 summarizes the levels of evidence of 34 articles selected from the 77 publications identified by the search strategy. No systematic reviews were identified for the use of dutasteride in the treatment of BPH. Most articles were of level 2 evidence, i.e. randomized controlled studies either reported in meeting abstracts or full publications. Four articles reported 2-year openlabel extension data from pooled randomized controlled studies and were classified as level 3 evidence. No articles were identified relating to pharmacoeconomic outcomes with dutasteride.
Table 1 | Evidence base included in the review

\begin{tabular}{|lcc|} 
& \multicolumn{2}{c|}{ Number of records } \\
\cline { 2 - 3 } Category & Full papers & Abstracts \\
\hline Initial search & 41 & 36 \\
records excluded & 31 & 12 \\
records included & 10 & 24 \\
Additional studies identified & 1 & 1 \\
Level 1 clinical evidence & 0 & 0 \\
Level 2 clinical evidence & 9 & 23 \\
Level $\geq 3$ clinical evidence & 2 & 2 \\
trials other than RCT & 0 & 0 \\
case studies & 0 & 0 \\
Economic evidence & 0 & 0 \\
\hline For definition of levels of evidence, see Editorial Information on inside back cover. \\
RCT, randomized controlled trials.
\end{tabular}

\section{Disease overview}

$\mathrm{BPH}$ is a common age-related condition in men. Clinical and histopathologic data suggest that $40-70 \%$ of men aged $60-70$ years are affected, and this rises to more than $80 \%$ in men over 80 years (Djavan 2002; Dull et al. 2002). Therefore, as the proportion of older people in the population grows then the prevalence of $\mathrm{BPH}$ is set to increase.

Although BPH is not a life-threatening disorder, bothersome LUTS can seriously affect patient quality of life. The condition is progressive and may lead to acute urinary retention (AUR), one of the most significant complications of long-term BPH. Analysis of placebo-treated groups from several multinational clinical trials has shown that prostate volume and serum prostate-specific antigen (PSA) levels are strong predictors of AUR in men with LUTS and clinically diagnosed BPH who were screened for prostate cancer (Roehrborn 2001; Roehrborn et al. 2001). It has been estimated that the incidence rate for AUR is between 5 and 25 per 1000 years (or 0.5-2.5\% per year) (Roehrborn 2001). The risk is cumulative and increases with age. This complication is one of the major factors that influences the need for surgery. Furthermore, these patients have a high risk of developing complications after prostatectomy. A prospective cohort study assessed complication rates and symptomatic outcomes in 3966 men undergoing prostatectomy (Pickard et al. 1998). When compared with those patients who underwent elective prostatectomy for alleviation of symptoms alone, men presenting with AUR had an excess risk of mortality and morbidity. Although the men with AUR were generally older, had larger prostate glands, and had more comorbidities, these factors did not fully explain the excess risk.

From both the patient and the economic points of view AUR and $\mathrm{BPH}$-related surgery are considered to be the most significant long-term risks of $\mathrm{BPH}$. A recent discrete choice study in the UK 
showed that the respondents were willing to wait 13,2 , and 8 months longer for symptom improvement in exchange for reduced prostate size, and an absolute 1\% decrease in the risks of AUR and surgery, respectively (Watson et al. 2004).

There are two components (one static, the other dynamic) of bladder outlet obstruction which are believed to contribute to LUTS. Smooth muscle tone in the bladder, prostatic urethra, prostate gland, and prostatic capsule is the dynamic component and accounts for up to $40 \%$ of the obstruction seen in BPH (de la Rosette et al. 2002). The static component of bladder outlet obstruction is prostate enlargement characterized by the increase in total volume and the transitional zone volume of the prostate gland. Medical treatments for BPH target either the dynamic or the static component of bladder outlet obstruction.

The pathophysiology of BPH is poorly understood. Nevertheless it is clear that proliferation of the prostatic tissue leading to enlargement is an age-dependent process which can restrict urine outflow. The principal stimulus for prostatic hyperplasia in later years is the androgen dihydrotestosterone (DHT). Testosterone is converted via 5AR to DHT and inhibition of this process leads to reduced levels of serum and intraprostatic DHT. Two isoforms (type 1 and type 2) of 5AR exist; type 2 is found in male genitalia and the prostate while type 1 is present in skin, liver, prostate, and kidney (Clark et al. 2004). DHT is essential for normal masculinization of the external genitalia and maturation of the prostate gland during development. In later life it is the principal androgen responsible for prostatic growth and so reducing serum DHT levels can result in a clinically measurable decrease in prostate volume over time through increased epithelial apoptosis. Thus inhibition of 5AR targets the static component (prostate enlargement) of bladder outlet obstruction.

Histologic changes in prostate tissue usually develop initially in men over 40 years and by the age of 60 years the prevalence of this change is more than $50 \%$ rising to $90 \%$ by 85 years (Roehrborn et al. 2003c). At present the best documented risk factors for $\mathrm{BPH}$ are age and functioning testes.

\section{Diagnosis of $\mathrm{BPH}$}

An early accurate diagnosis of BPH is important for determining the most appropriate treatment option and maximizing the treatment outcomes. The presence of LUTS is not specific or exclusive to $\mathrm{BPH}$ and it is important to exclude other conditions which may be responsible for these symptoms. In addition, the presence of LUTS does not necessarily indicate an enlarged prostate and BPH can also exist in the absence of LUTS.

Clinical practice guidelines for the diagnosis and management of $\mathrm{BPH}$ have been produced and recently updated in the USA (Roehrborn et al. 2003c) and Europe (Madersbacher et al. 2004). Most guidelines stipulate that an initial evaluation should involve taking a detailed medical history focusing on the urinary tract coupled with a digital rectal examination (DRE) of the prostate. Other recommended tests include uroflowmetry
Table 2 | Categories of symptom severity described by the International Prostate Symptom Score (I-PSS)

\begin{tabular}{|ll|}
\hline Symptom score & Symptom severity \\
\hline $0-7$ & Mild \\
$8-19$ & Moderate \\
$20-35$ & Severe \\
\hline
\end{tabular}

[including volume of postvoid residual (PVR) urine], urinalysis, measurement of creatinine and serum PSA, and symptom assessment with a validated questionnaire. A questionnaire commonly used to assess and quantify patient symptoms is the International Prostate Symptom Score (I-PSS) which consists of eight questions; seven questions investigate urinary symptoms and one explores the quality of life [the American Urological Association (AUA)-Symptom Index; AUA-SI is equivalent to the first seven questions of the I-PSS, but does not assess quality of life]. When compared with an informal discussion this instrument provides a superior assessment of symptom severity and frequency. Completion of the assessment provides a classification of disease severity ranging from mild, moderate, or severe (Table 2).

Taken together, the various recommended diagnostic assessments are designed to indicate or exclude the presence of prostate cancer, estimate the size of the prostate gland, and establish the severity and degree of bother caused by BPH symptoms. The uroflowmetry studies are considered to be the best noninvasive urodynamic tests to determine obstruction of the lower urinary tract.

\section{Current therapy options}

The aim of treating BPH is to relieve LUTS and reduce the risk of AUR and BPH-related surgery which all affect patient quality of life. Factors that need to be taken into account when considering treatment options include the severity of symptoms, prostate size, and patient preferences. There are three courses of action for the management of this condition: watchful waiting, medical therapy, and surgical or minimally invasive interventions. Updated treatment guidelines based on recent clinical data with these options have been published (Roehrborn et al. 2003c; Madersbacher et al. 2004). Medical therapies are summarized in Table 3.

\section{Watchful waiting}

Watchful waiting is recommended for patients with mild symptom scores (typically $\leq 7$; see Table 2 ) that do not affect daily life activities. Although the patient receives no active treatment for $\mathrm{BPH}$, simple changes in lifestyle such as decreasing alcohol and caffeine intake, and fluid intake before bedtime may reduce bothersome symptoms (Madersbacher et al. 2004). It is vital that patients managed with watchful waiting are regularly followed up by their physician to monitor any progression (or remission) of the condition. 
Table 3 | Summary of medical options for the treatment of benign prostatic hyperplasia

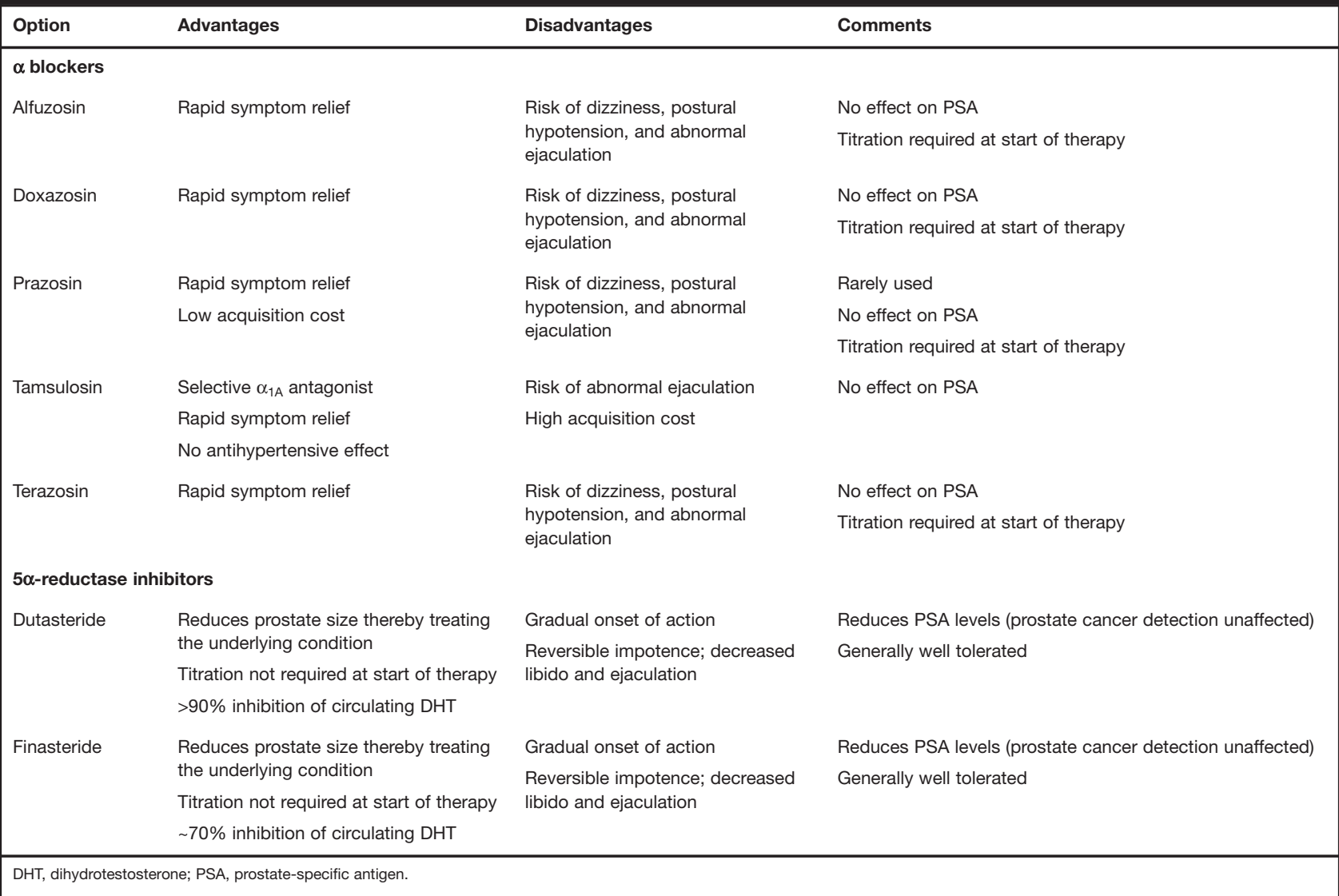

\section{Medical therapy}

Medical therapies can provide significant relief from symptoms with less trauma compared with surgical treatment. Thus medical therapies are an appropriate option for patients with uncomplicated BPH with moderate to severe symptoms.

The alfa blockers (alfa ${ }_{1}$-adrenoceptor antagonists), such as doxazosin, relieve the dynamic component of bladder outlet obstruction through their effect on blocking alfa $_{1}$-adrenoceptormediated contraction of prostatic smooth muscle and the bladder neck. These agents can improve BPH symptoms rapidly (within 2-4 weeks) and this also allows the treatment to be evaluated and modified relatively quickly. Although use of an alfa blocker can delay BPH-related surgery and the incidence of AUR, risk of these events still occurring has been shown to remain unaltered after 4.5 years treatment with doxazosin (McConnell et al. 2003). It remains to be determined whether or not this is true for the newer alfa blockers. Side effects encountered with these agents include fatigue, asthenia, ejaculatory problems, and postural hypotension. These can affect a significant number of patients; however, they may be minimized by slow titration and by bedtime administration. In order to minimize the side effects of the nonselective alfa blockers (e.g. doxazosin, prazosin, and terazosin) a highly selective alfa $\mathrm{A}$-adrenoceptor antagonist, tamsulosin, has been developed. Although it is not associated with hypotension it is more costly compared with the nonselective alfa blockers.

The 5ARls target prostate enlargement which is the static component of bladder outlet obstruction. There are two 5ARIs currently available, finasteride and dutasteride. Finasteride selectively inhibits type $25 \mathrm{AR}$ at therapeutic doses while dutasteride inhibits both type 1 and type 2 isoforms (Clark et al. 2004). In theory the greater suppression of DHT achieved from inhibition of both 5AR isoforms could result in greater efficacy than that with just selective type 2 inhibition as circulating DHT generated by peripherally type $15 \mathrm{AR}$ may still influence prostate growth (Bramson et al. 1997).

Finasteride was the first 5ARI introduced for the treatment of $\mathrm{BPH}$. During a 4-year placebo-controlled study finasteride treatment reduced the risk of AUR and the need for surgery by more than $50 \%$, and significantly improved symptom scores, improved urinary flow rates, and reduced prostate volume (all $P<0.001$ ) compared with placebo (McConnell et al. 1998). Side effects are associated with sexual function and include erectile and ejaculatory dysfunction, and decreased libido; however, these are reversible on cessation of treatment and the incidence of newly reported events decreases after the first year. 
Finasteride treatment also lowers serum PSA levels by about $50 \%$. In patients treated with finasteride, serum PSA levels should be multiplied by 2 (the "doubling rule") and then compared with the normal ranges for untreated men. Data from the PLESS (Proscar Long-term Efficacy and Safety Study) trial involving 3040 men have demonstrated that this approach preserves the value of PSA as a tool for prostate cancer detection (by prompting prostate biopsy for further investigation) (Andriole et al. 1998).

The attributes of a 5ARI (dutasteride) and alfa blockers were investigated in a discrete choice study in community dwelling men (>40 years) in the UK (Watson et al. 2004). Using willingness to pay as a measure of preference for the defined drug profiles, respondents were worse off (by almost $£ 12$ monthly) if they received alfa blockers compared with no medication. In contrast, if respondents were on 5ARIs they were better off by $£ 19$, demonstrating a preference for the attributes of this treatment.

The combination of an alfa blocker and 5ARI in a 4.5-year study has been shown to be more effective in preventing the progression of BPH than the single therapies (McConnell et al. 2003). In this study, the National Institutes of Health (NIH) Medical Therapy of Prostatic Symptoms (MTOPS) trial, overall progression was defined as a $\geq 4$ point increase in AUA-SI score, AUR, urinary incontinence, renal insufficiency, or recurrent urinary tract infection. When compared with placebo treatment, the combination drug regimen reduced the risk of overall BPH progression by $67 \%$, compared with $39 \%$ for doxazosin alone and $34 \%$ for finasteride alone $(P<0.001$ for each pairwise comparison of combination therapy vs monotherapy). Symptom scores were significantly improved by combination therapy, finasteride, or doxazosin $(P<0.001$ for all treatments); combination therapy was superior to both doxazosin $(P=0.006)$ and finasteride $(P<0.001)$ alone. However, the risks of AUR and the need for BPH-related surgery were significantly reduced by combination therapy and finasteride $(P<0.001$ for both treatments), but not by doxazosin (McConnell et al. 2003).

\section{Surgical treatment}

The most well-established treatment option for BPH is surgery. This intervention is recommended in patients with complicated $\mathrm{BPH}$ or if previous medical treatments have failed, leaving the patient with bothersome voiding symptoms such that their quality of life is seriously affected. Transurethral resection of the prostate (TURP) has become a well-established standard treatment for $\mathrm{BPH}$. However, surgery can be associated with significant shortand long-term morbidity (bleeding, urinary tract infection, retrograde ejaculation, and urethral strictures), and repeated treatment may be necessary in up to $5-15 \%$ of patients within 8 years (Zlotta \& Djavan 2002). In addition, TURP may not be a suitable treatment for some patients who wish to avoid an invasive surgical procedure or for whom surgery may pose excessive risks. These factors have led to a number of recent developments aimed at achieving the benefits of TURP but at lower costs and with less morbidity. Thus minimally invasive therapy (using thermal energy sources to produce coagulation necrosis of prostate tissue) and surgical endoscopic techniques are now available.

Minimally invasive therapies may reduce the need for general anesthesia and are associated with a lower rate of repeat procedure; nevertheless they do not necessarily reach the same levels of symptom improvement as TURP. In fact these therapies have often been evaluated in comparison with TURP, but it is perhaps more appropriate that they should be compared against medical therapies which are usually taken for long periods and which reduce in part or delay the need for surgery.

Other treatment options including transurethral needle ablation (TUNA), transrectal high-intensity focused ultrasound, and prostate stents have not been adequately assessed for long-term benefits.

\section{Clinical evidence with dutasteride in $\mathrm{BPH}$}

Most clinical trial data with dutasteride have been derived from three parallel, randomized, placebo-controlled studies of 2 years' duration [ARIA3001 (US only), ARIA3002 (US only), and ARIB3003 (19 countries)] involving 400 participating centers and 4325 patients. Results from these studies have been published and the data have also been pooled as the inclusion and exclusion criteria for the trials were identical. All three studies have continued with 2-year open-label extensions during which all patients received dutasteride, thus providing up to 4 years of observational data. Results from these studies have been published extensively in meeting abstracts and full publications.

These trials evaluated patient-oriented outcomes (such as symptom relief, reduction in the risks of AUR and surgery), and measurement of disease-oriented surrogate markers (e.g. serum DHT levels), safety, and tolerability. Patient-oriented outcomes are by definition not only important and relevant to patients but also to clinicians for everyday practice.

Two studies have directly compared dutasteride with finasteride (Clark et al. 2004; Hagerty et al. 2004). In addition, a combination study (SMART-1) with the alfa blocker tamsulosin has also been conducted (Barkin et al. 2003). A large multinational 4-year randomized study, COMBAT [Combination of Avodart ${ }^{\circledR}$ (dutasteride) And Tamsulosin] is underway and is expected to elucidate the effects of combination therapy for patients with symptomatic BPH at high risk of disease progression (Kaplan 2004). Dutasteride has not been compared against an alfa blocker for the treatment of BPH in any studies identified.

\section{Reduction in the risk of AUR and BPH-related surgery}

Pooled results from the ARIA3001, ARIA3002, and ARIB3003 randomized controlled trials provide strong evidence that both the incidence of surgical interventions and episodes of AUR are reduced by dutasteride treatment (Table 4). At the end of the 2year double-blind phase of these studies, the reduction in risk of 


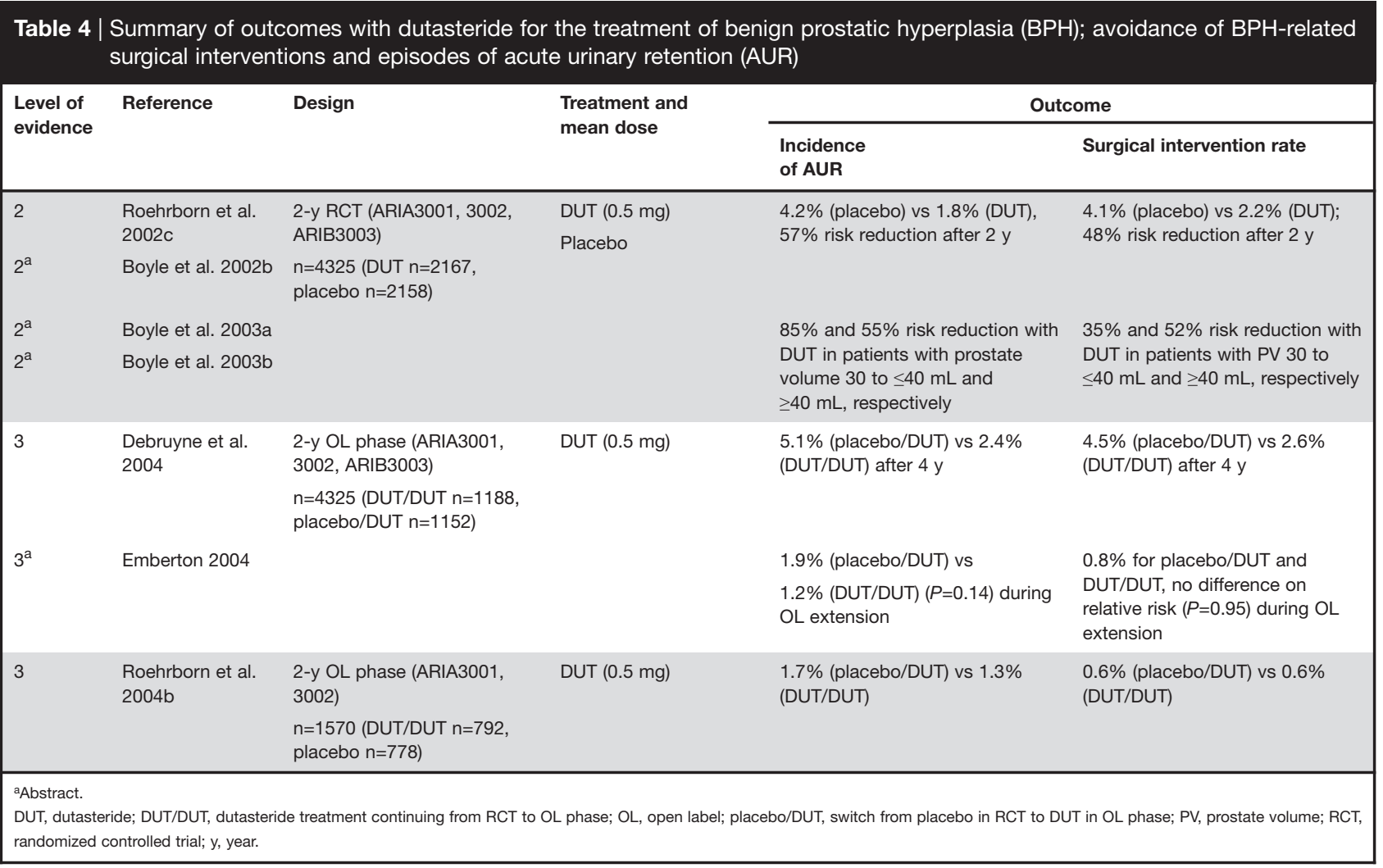

$\mathrm{BPH}$-related surgical intervention was $48 \%$ and the risk reduction of AUR was $57 \%$ compared with placebo (Roehrborn et al. 2002c). This effect was maintained for a further 2 years of observation, representing good level 3 evidence. Although the 2year extensions were not placebo controlled, the low rates of surgical or AUR events suggest the benefit in attenuating the progression of $\mathrm{BPH}$ with dutasteride treatment for up to 4 years.

\section{Symptom relief}

There is evidence from pooled data from three studies that dutasteride provides relief from the symptoms of BPH (Table 5). Symptom relief has been measured by improvements in patientreported AUA-SI and BPH-Impact Index (BII) scores and clinically measured uroflowmetry $\left(Q_{\max }\right)$.

In the pooled studies AUA-SI scores improved significantly compared with placebo $(P<0.001)$ from 6 months onwards, but a change in baseline was demonstrated as early as 3 months in one study (Roehrborn et al. 2002c). After 4 years' treatment with dutasteride AUA-SI improved by 6.5 points compared with baseline (Debruyne et al. 2004). Not only is this a statistically significant improvement compared with baseline $(P<0.001)$ and placebo $(P<0.001)$ treatment, but this change is also in excess of that defined as a clinically relevant decrease in symptoms $(\geq 3.1$ points) and one that would be perceptible to patients (Barry et al. 1995).

$Q_{\max }$ improved with dutasteride and significant differences
$(P \leq 0.006)$ compared with placebo were seen as early as 1 month after starting treatment and at all measured endpoints up to 2 years. In each of the three individual studies the effect on $Q_{\max }$ became statistically significant after 3 months' treatment with dutasteride compared with placebo (Roehrborn et al. 2002c).

A comparison of dutasteride and finasteride (doses not stated) on symptom relief has been published as a recent meeting abstract. This single-center 3-month prospective study recruited 240 consecutive men with benign prostatic enlargement and symptomatic BPH who were assigned to dutasteride $(n=120)$ or finasteride $(n=120)$ (Hagerty et al. 2004). Patients receiving dutasteride had a greater reduction in AUA-SI scores compared with those receiving finasteride: 36 vs 22 had a 1-point improvement, 14 vs five had a 2-point improvement, two vs one had a 3-point improvement, and 68 vs 92 had no change in their score. The estimated difference between the two treatment groups for the proportion of subjects with an improvement in AUA-SI score was 20\% (95\% confidence intervals $7-32.5 \%$; $P<0.0016)$. Although the magnitude of response for dutasteride was greater than finasteride it is unclear whether this is due to a difference in time of onset of action.

EPICS (Enlarged Prostate International Comparator Study) was a short-term direct comparator trial between dutasteride $(0.5 \mathrm{mg})$ and finasteride (5 mg) in men with BPH (Gilling et al. 2005). Following a 4-week placebo run-in period, 1630 patients (eligibility; age >50 years, AUA-SI $\geq 12$, prostate volume $\geq 30 \mathrm{~mL}$, PSA $1.5-10 \mathrm{ng} / \mathrm{mL}$, 
$\left.Q_{\max } \leq 15 \mathrm{~mL} / \mathrm{s}\right)$ were randomized to dutasteride $(\mathrm{n}=813)$ or finasteride $(n=817)$ for 48 weeks. After 1 year similar improvements from baseline in AUA-SI of 6.2 and 5.8 and in $Q_{\max }$ of 2.1 and $1.8 \mathrm{~mL} / \mathrm{s}$ were seen with dutasteride and finasteride, respectively. Given the gradual, progressive course of $\mathrm{BPH}$ and the mode of action of 5ARIs major differences in efficacy would not necessarily be seen in studies of up to 1-year duration (Gilling et al. 2005).

There may be a delay of up to 6 months before 5ARIs improve symptoms. In contrast, the onset of symptom relief with alfa blockers is more rapid. The SMART- 1 study $(n=327$ patients with moderate to severe urinary symptoms, IPSS $\geq 12$ ) was initiated to investigate speed of symptom relief with tamsulosin combined with dutasteride, then maintenance of effect following withdrawal of the alfa blocker after 6 months (Barkin et al. 2003). There is evidence that early symptom relief was achieved with this combination, with $66 \%$ of men reporting improvement after 4 weeks. The study also showed that tamsulosin could be withdrawn from the majority of men $(77 \%)$ after 6 months with maintenance or improvement of symptom relief. Of the subjects with moderate baseline symptoms (IPSS <20), 84\% switched to dutasteride monotherapy with no noticeable deterioration in their symptoms. A subgroup of patients (27\%) with severe baseline symptoms (IPSS $\geq 20$ ) reported a higher rate of worsening of symptoms when tamsulosin was withdrawn compared with patients with moderate symptoms (42.5\% vs $16 \%)$. Thus patients with severe symptoms may benefit from longer combination therapy but the study did not continue beyond 6 months to allow this to be explored.

\section{Reduction in androgen levels and prostate gland size}

There is good evidence that dutasteride reduces the diseaseoriented outcome of prostate volume in men with $\mathrm{BPH}$, as measured by total prostate volume (TPV) and transition zone volume (TZV) (Table 6). Reductions in mean TPV were evident after 1 month, with further shrinkage as dutasteride treatment continued, by up to $26.2 \%$ after 4 years (Roehrborn et al. 2004b). An abstract report of these studies showed that, at 2 years, the effect of dutasteride in reducing prostate volume was equal for patients with smaller ( 30 to $\leq 40 \mathrm{~mL}$ ) compared with larger baseline prostate volume $(\geq 40 \mathrm{~mL}$ ) (Boyle et al. 2003a). These data suggest that dutasteride may be initiated early in the time course of BPH progression in order to limit prostate growth.

As discussed above, DHT is the principal androgen responsible for stimulating prostatic growth. Dutasteride has been shown to effectively reduce serum levels of DHT in a number of large studies. The onset of suppression of DHT is rapid and $\geq 90 \%$ reduction has been seen in $58 \%$ of patients after 1 month's treatment with dutasteride (Roehrborn et al. 2002c). After 4 years' treatment with dutasteride, serum DHT levels were reduced by $\geq 90 \%$ in $87 \%$ of patients (Debruyne et al. 2004).

Dutasteride has also been shown to be more effective at suppressing serum DHT levels compared with finasteride (Clark et al. 2004). After 24 weeks of treatment, suppression of DHT was statistically significantly greater with $0.5 \mathrm{mg}$ dutasteride $(94.7 \%$ inhibition) compared with $5 \mathrm{mg}$ finasteride $(70.8 \%$ inhibition, $P<0.001)$. A greater proportion of dutasteride-treated patients also achieved suppression of DHT levels by both $>70 \%$ and $>90 \%$ compared with finasteride-treated patients (Roehrborn et al. 2003a). These data demonstrate that dutasteride reduces circulating levels of $\mathrm{DHT}$ to a greater extent than finasteride and that maximal reduction of DHT is achieved in a larger proportion of patients thus minimizing the influence of this androgen on prostate growth.

As well as reducing DHT levels in serum, dutasteride has been shown to reduce intraprostatic levels of the androgen which is important in the context of the underlying disease pathophysiology. In a small randomized double-blind study 46 patients with biopsy-proven T1/T2 prostate cancer were treated with placebo or dutasteride for up to 82 days prior to radical prostatectomy (Andriole et al. 2003). Dutasteride powerfully suppressed both serum and intraprostatic DHT by $96.7 \%$ and $>97 \%$ respectively, compared with baseline and placebo treatment $(P<0.001)$. However, it should be noted that the dose of dutasteride $(5 \mathrm{mg}$ ) used in this study was 10 -fold greater than the recommended therapeutic dose.

\section{Effects on PSA levels}

Dutasteride treatment for 4 years resulted in the reduction in serum PSA levels from baseline by $57 \%$ (Table 7 ). Most of this reduction occurred within 1 year of treatment (Andriole \& Kirby 2003). One concern with reducing PSA levels is the potential masking of prostate cancer detection. Data from the 4-year pooled analysis showed that $77 \%$ of the dutasteride-treated patients with prostate cancer reported during the study had an increase in PSA after nadir compared with $52 \%$ of patients with no diagnosis of prostate cancer. Among dutasteride-treated patients with no diagnosis of prostate cancer the median maximum increase in PSA from nadir was $0.1 \mathrm{ng} / \mathrm{mL}$ compared with $0.9 \mathrm{ng} / \mathrm{mL}$ in patients with a diagnosis of prostate cancer. Monitoring PSA over time therefore allows a risk of prostate cancer to be assessed (Roehrborn et al. 2004a). In addition, dutasteride had no clinically meaningful effect on the free-to-total (F/T) PSA ratio. Thus the utility of the sensitivity and specificity of $\mathrm{F} / \mathrm{T}$ PSA is maintained as a screen for detecting prostate cancer in men treated with a 5ARI (Andriole et al. 2002).

PSA values should be doubled for patients who have been treated with dutasteride for at least 6 months to determine whether or not isolated PSA levels fall within normal ranges in untreated men (Andriole \& Kirby 2003). As has been shown with finasteride, use of the "doubling rule" preserves the usefulness of PSA for detecting prostate cancer (Andriole et al. 1998).

\section{Safety and tolerability}

Results from observational studies provide good evidence of the safety and tolerability of dutasteride in a large number of patients treated over a long period of up to 4 years (Roehrborn et al. 2002c, 2004b; Andriole \& Kirby 2003; Debruyne et al. 2004) (Table 8). In addition, up to 1 year's comparative data with dutasteride and finasteride have been obtained from two studies (ARI40001 


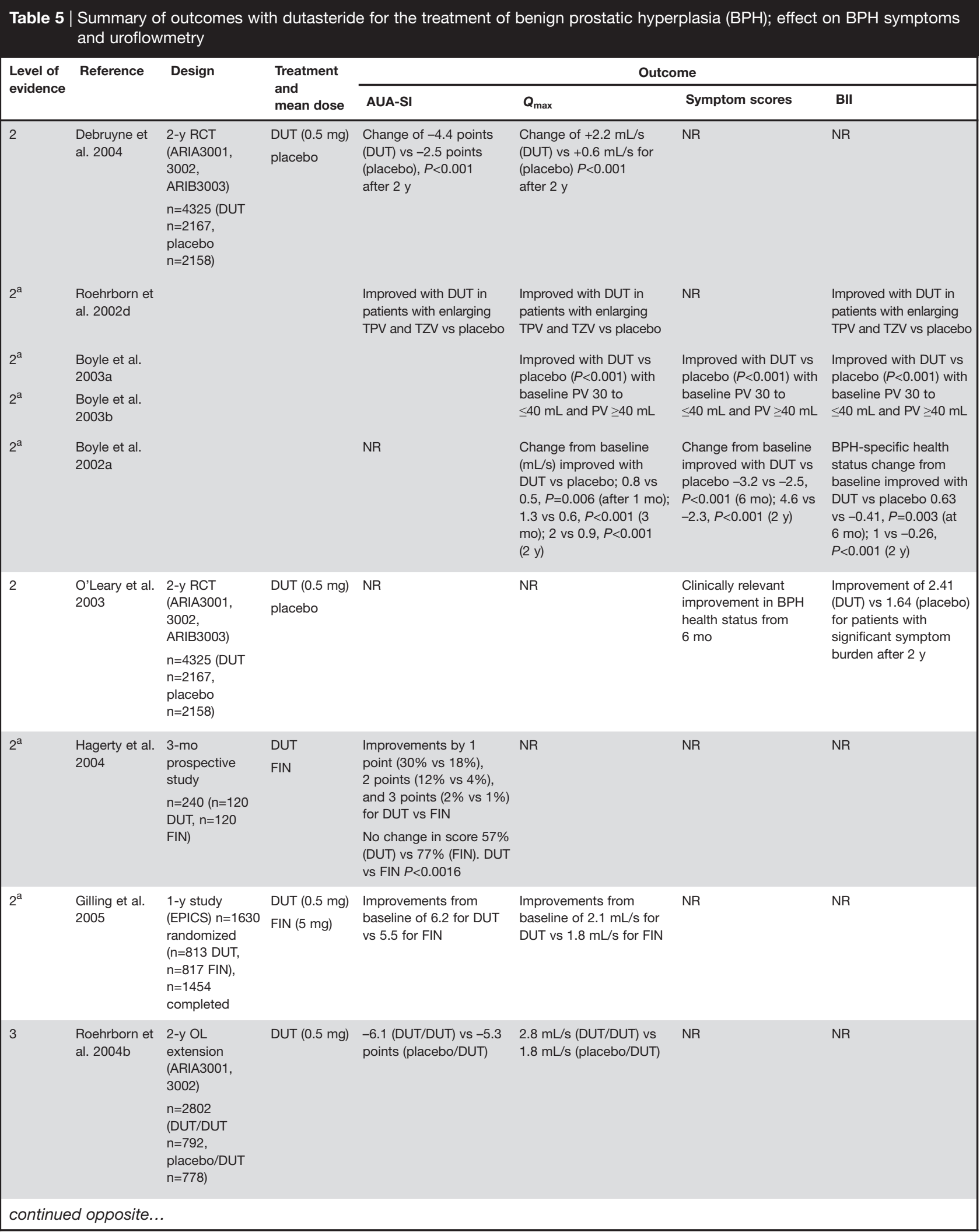


Dutasteride | clinical impact review

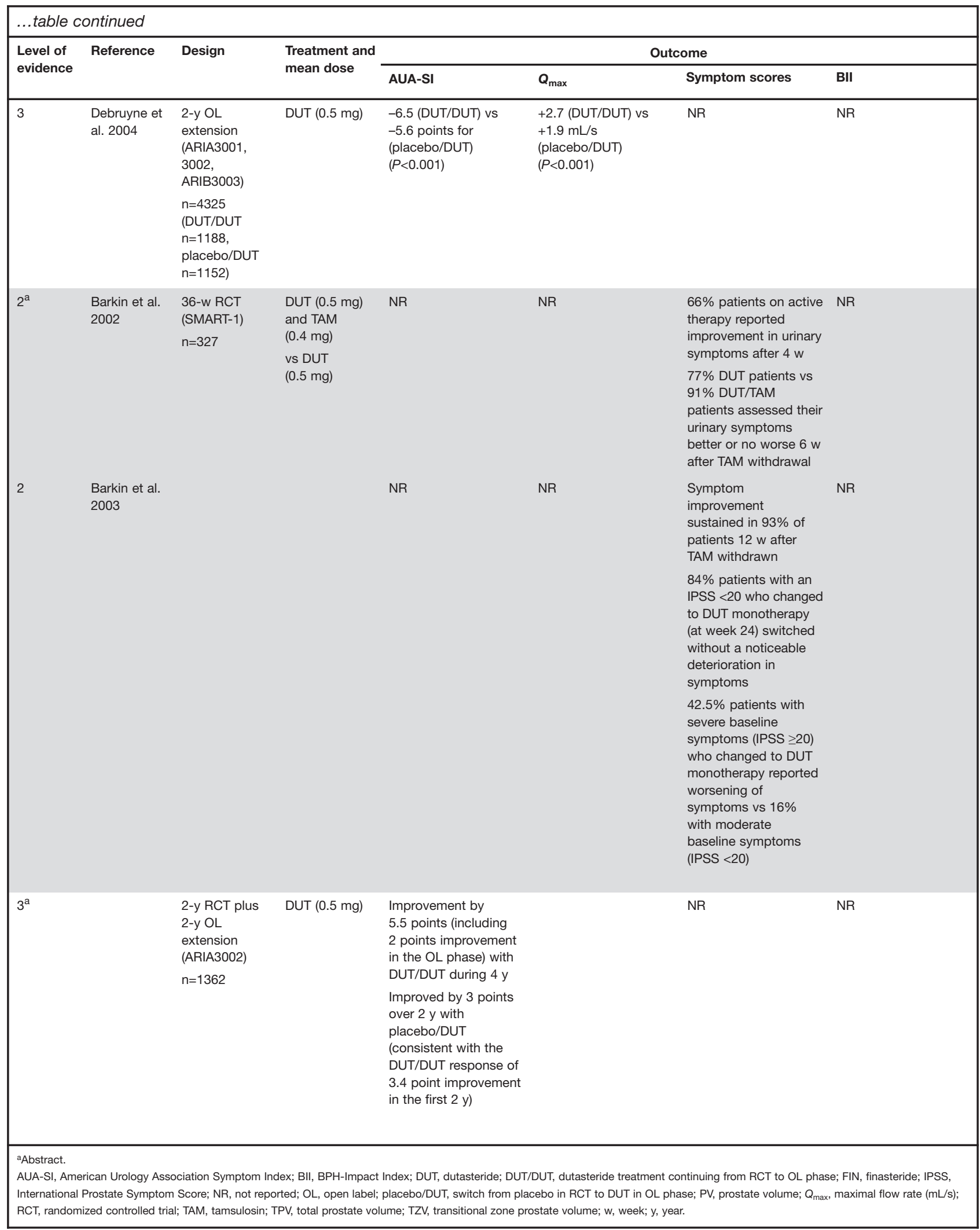




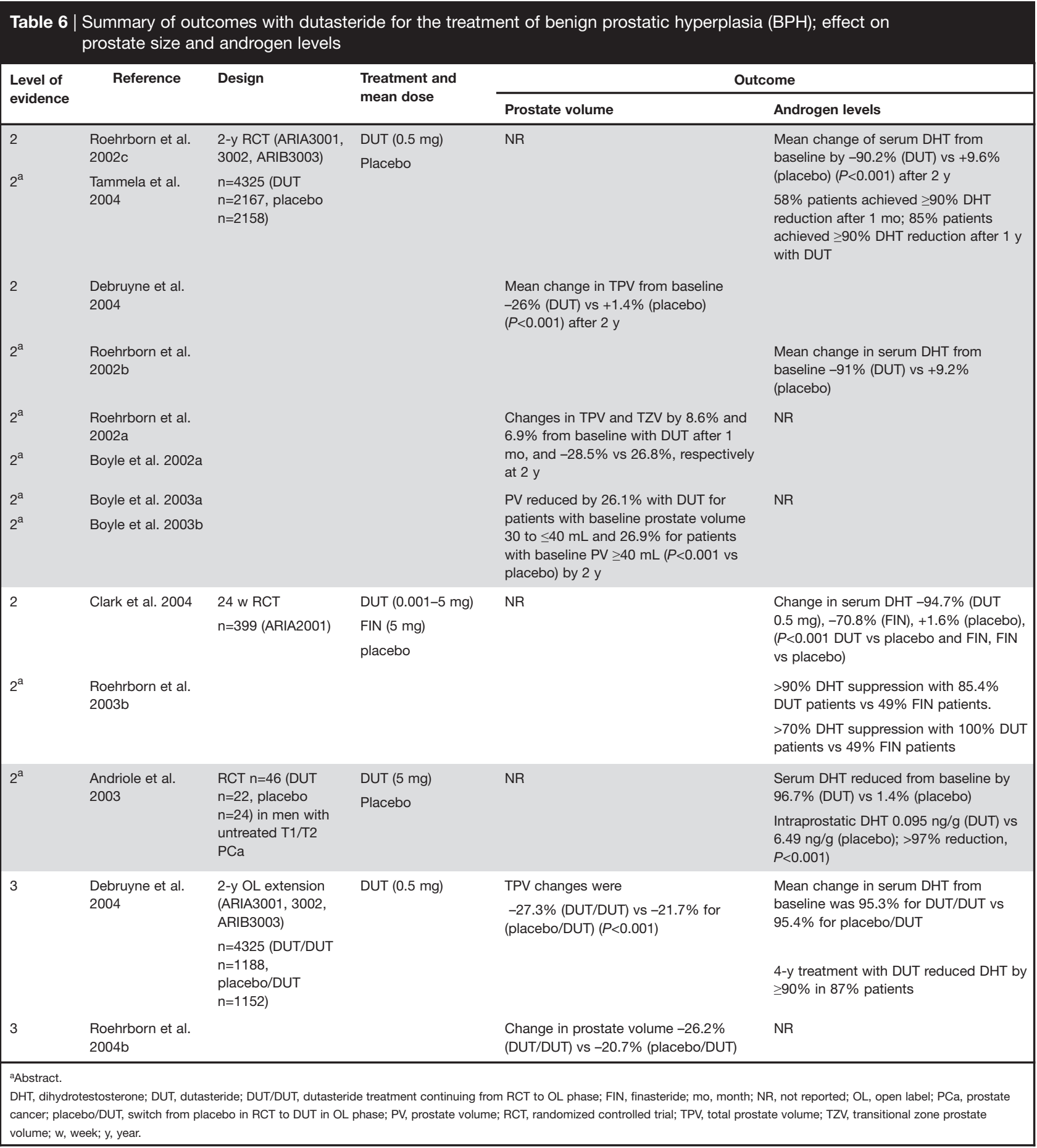

1-year duration, and ARIA2001, a 24-week study) (Andriole \& Kirby 2003; Clark et al. 2004).

These studies show that the incidence of general adverse events with dutasteride was similar to that with placebo. The exception was an elevated incidence of sexually-related adverse events which were seen more commonly with dutasteride in the first 6 months of treatment compared with placebo; impotence 4.7 vs $1.7 \%(P<0.05)$, decreased libido 3 vs $1.4 \%(P<0.05)$, ejaculation disorder 1.4 vs $0.5 \%(P<0.05)$, and gynecomastia 0.5 vs $0.2 \%$ (not significant). The incidence of these events (except impotence, 1.4 vs $1.5 \%$ for dutasteride 
Table 7 | Summary of the outcomes associated with dutasteride and prostate-specific antigen (PSA)

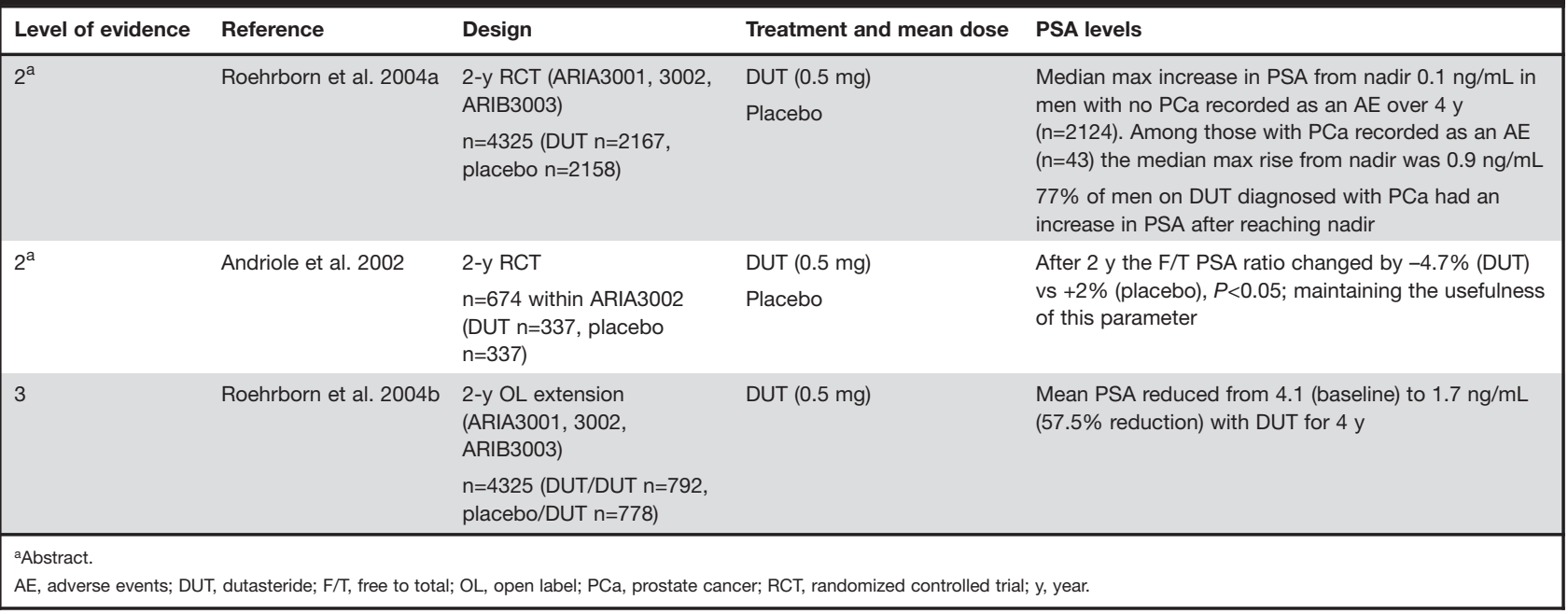

and placebo, respectively) was $<1 \%$ after 1 year's treatment suggesting that they were transient and reversible (Andriole \& Kirby 2003).

The adverse event profile with clinically used doses of dutasteride $(0.5 \mathrm{mg} /$ day) and finasteride (5 mg/day) were similar (Clark et al. 2004). There were no significant differences in the incidence of the most common adverse events between the active treatment groups or placebo, other than an increased reporting of altered libido among patients receiving finasteride or dutasteride ( $\geq 0.5 \mathrm{mg} /$ day).

\section{Resource utilization}

Medical treatment for $\mathrm{BPH}$ requires lifetime medication. In comparison, surgery is generally a one-off event which can be costly due to the requirement for a stay in the hospital. It has been estimated that the 5-year cost of treatment of BPH by TURP is \$US7334 compared with typical drug therapy costing \$US6294 during the same time-period (Dull et al. 2002). A patient's drug treatment costs are likely to be stable but if a repeat procedure is required then the cost of surgery effectively doubles.

Because they alter the natural history of symptomatic BPH the 5ARIs are the only medical treatment for $\mathrm{BPH}$ that reduce prostate volume, which may influence the need for surgery and reduce the risk of AUR. Data obtained with dutasteride have provided evidence that indicates both surgery and AUR can be avoided. AUR is one of the most important complications with $\mathrm{BPH}$. At the very least the patient will present in pain with the inability to urinate and will probably require catheterization in the emergency room. Follow-up visits, catheter removal, and then surgery are the likely costly sequelae of AUR. Clearly a treatment that ultimately avoids, rather than just delays, the need for managing AUR and surgery can have important implications in resource utilization. Hospital resources are under greater pressure as the population ages and so any treatment that can reduce this burden is appealing. Furthermore, reducing the need for TURP would have significant implications as it is the second most common surgical procedure (after cataract extraction) in men older than 65 years with 400000 operations costing \$US5 billion per year (Oesterling 1995).

Although dutasteride is effective in reducing the signs and symptoms of BPH there is a delay in the onset of statistically significant symptom relief of at least 3 months (Roehrborn et al. 2002c). This delay may be reduced by the short-term combination of dutasteride with an alfa blocker. So far only the effects of combining tamsulosin with dutasteride have been reported (Barkin et al. 2002, 2003). Nevertheless it is possible that other alfa blockers may be used in combination. For example, doxazosin has been used in combination with finasteride for the generation of rapid symptom relief. The AUA guidelines note that the combination of an alfa blocker and a $5 \mathrm{ARI}$ is an appropriate and effective treatment for patients with LUTS associated with demonstrable prostatic enlargement (Roehrborn et al. 2003c). The cost of adding an extra treatment to the patient's medication has obvious implications to budgets and resources.

The optimal period for continuing with an alfa blocker in combination therapy is unclear. Whether it can be withdrawn after short-term symptom relief has been achieved or continued treatment is necessary remains to be determined. It is also unclear as to whether or not combination therapy would lead to improved compliance with drug treatment due to patients perceiving improvements in treatment effects.

\section{Patient group/population}

Dutasteride and finasteride have been recommended as appropriate and effective treatments for patients with LUTS associated with demonstrable prostatic enlargement (Roehrborn et al. 2003c). This is because they affect the natural history of the 
Table 8 | Summary of outcomes with dutasteride for the treatment of benign prostatic hyperplasia (BPH); safety and tolerability

\begin{tabular}{|c|c|c|c|c|}
\hline $\begin{array}{l}\text { Level of } \\
\text { evidence }\end{array}$ & Reference & Design & $\begin{array}{l}\text { Treatment and } \\
\text { mean dose }\end{array}$ & Incidence of adverse events \\
\hline 2 & $\begin{array}{l}\text { Andriole \& } \\
\text { Kirby } 2003\end{array}$ & $\begin{array}{l}\text { 2-y RCT (ARIA3001, } \\
\text { 3002, ARIB3003) } \\
n=4325 \text { (DUT } n=2167 \text {, } \\
\text { placebo } n=2158)\end{array}$ & $\begin{array}{l}\text { DUT }(0.5 \mathrm{mg}) \\
\text { Placebo }\end{array}$ & $\begin{array}{l}6 \text {-mo results: sexual AEs were more common with DUT vs placebo (impotence } 4.7 \% \text { vs } \\
1.7 \%, P<0.05 \text {; decreased libido } 3 \% \text { vs } 1.4 \%, P<0.05 ; \text { gynecomastia } 0.5 \% \text { vs } 0.2 \%, N S \text {; } \\
\text { ejaculation disorder } 1.4 \% \text { vs } 0.5 \%, P<0.05 \text { ) } \\
2 \text {-y results: sexual AEs with DUT vs placebo (impotence } 0.8 \% \text { vs } 0.9 \% \text {, NS; decreased } \\
\text { libido } 0.3 \% \text { vs } 0 \% \text {, NS; gynecomastia } 0.6 \% \text { vs } 0.1 \%, P<0.05 \text {; ejaculation disorder } 0.1 \% \\
\text { vs } 0 \%, \text { NS) }\end{array}$ \\
\hline $2^{a}$ & $\begin{array}{l}\text { Carson et } \\
\text { al. } 2003\end{array}$ & & & $\begin{array}{l}\text { Maximal suppression of DHT with DUT does not increase the incidence of drug-related } \\
\text { sexual AEs and gynecomastia over that historically reported for other } 5 \text { AR inhibitors }\end{array}$ \\
\hline 2 & $\begin{array}{l}\text { Clark et al. } \\
2004\end{array}$ & $\begin{array}{l}24 \mathrm{w} \text { RCT } \\
\mathrm{n}=399 \text { (ARIA2001) }\end{array}$ & $\begin{array}{l}\text { DUT (0.01-5 mg) } \\
\text { FIN }(5 \mathrm{mg}) \\
\text { Placebo }\end{array}$ & $\begin{array}{l}\text { Altered libido in } 2 \% \text { (placebo), } 11 \% \text { (DUT } 0.5 \mathrm{mg} \text { ), } 13 \% \text { (FIN) }(P<0.05 \text { vs placebo) } \\
\text { patients; impotence in } 3 \% \text { (placebo), } 5 \% \text { (DUT) and } 11 \% \text { (FIN) patients }\end{array}$ \\
\hline $2^{a}$ & $\begin{array}{l}\text { Clark et al. } \\
2003 \\
\text { Clark \& } \\
\text { Matsumoto } \\
2003\end{array}$ & RCT $n=99$ & $\begin{array}{l}\text { DUT }(0.5 \mathrm{mg}) \\
\text { FIN }(5 \mathrm{mg}) \\
\text { placebo }\end{array}$ & $\begin{array}{l}\text { No clinically significant changes from baseline in bone density, markers of bone } \\
\text { metabolism or lipid levels between treatment groups for }<1 \text { y and follow-up weeks } 20-24\end{array}$ \\
\hline 2 & $\begin{array}{l}\text { Andriole \& } \\
\text { Kirby } 2003\end{array}$ & $\begin{array}{l}\text { 36-w RCT (SMART-1) } \\
\mathrm{n}=327\end{array}$ & $\begin{array}{l}\text { DUT }(0.5 \mathrm{mg}) \text { and } \\
\text { TAM }(0.4 \mathrm{mg}) \text { vs } \\
\text { DUT }(0.5 \mathrm{mg})\end{array}$ & $\begin{array}{l}\text { Ejaculation disorders were the only AEs considered to be at least possibly drug related } \\
\text { and occurring in } \geq 5 \% \text { of patients }\end{array}$ \\
\hline 3 & $\begin{array}{l}\text { Roehrborn } \\
\text { et al. } 2004 \mathrm{~b}\end{array}$ & $\begin{array}{l}\text { 2-y OL extension } \\
\text { (ARIA3001, 3002) } \\
\mathrm{n}=2802 \text { (DUT/DUT } \\
\mathrm{n}=792, \text { placebo/DUT } \\
\mathrm{n}=778 \text { ) }\end{array}$ & DUT (0.5 mg) & $\begin{array}{l}1 \text {-y results: sexual AEs with DUT/DUT vs placebo/DUT (impotence } 1.6 \% \text { vs } 3.1 \% \text {, } \\
\text { decreased libido } 0.5 \% \text { vs } 2.6 \% \text {, gynecomastia } 1.9 \% \text { vs } 1.5 \% \text {, ejaculation disorder } 0.3 \% \\
\text { vs } 1.3 \% \text { ) } \\
2-y \text { results: sexual AEs with DUT/DUT vs placebo/DUT (impotence } 0.6 \% \text { vs } 0.4 \% \text {, } \\
\text { decreased libido } 0 \% \text { vs } 0.3 \% \text {, gynecomastia } 1 \% \text { vs } 1.1 \% \text {, ejaculation disorder } 0.5 \% \text { vs } \\
0.1 \% \text { ) }\end{array}$ \\
\hline
\end{tabular}

aAbstract.

5AR, 5-alfa-reductase; AE, adverse event; DHT, dihydrotestosterone; DUT, dutasteride; DUT/DUT, dutasteride treatment continuing from RCT to OL phase; FIN, finasteride; mo, month; NS, not significant; OL, open label; placebo/DUT, switch from placebo in RCT to DUT in OL phase; RCT, randomized controlled trial; TAM, tamsulosin; w, week; y, year.

disease and affect its progression by limiting prostate enlargement. The benefits of this may be seen in the reduced occurrence of AUR and avoidance of surgery.

Because 5ARls are not as effective as alfa blockers (and have a slower onset of action) for symptom relief they are not recommended for the treatment of LUTS alone in men with no prostatic enlargement. In this case alfa blockers are an appropriate treatment option to manage troublesome symptoms through the dynamic component of bladder outlet obstruction.

Prostate volume is reduced by $18-27 \%$ in men receiving 5 ARIs for up to 4 years, irrespective of baseline prostate volume (McConnell et al. 1998; Roehrborn et al. 2004b). Early initiation of a 5ARI in symptomatic men with enlarged prostates and a PSA level of $\geq 1.5 \mathrm{ng} / \mathrm{mL}$ has been proposed as a reasonable approach to decreasing prostate volume and maintaining the reduction long term (Roehrborn et al. 2004b).
In addition to the demonstrable presence of an enlarged prostate gland other factors may influence the choice of dutasteride for the treatment of $\mathrm{BPH}$ for specific patient groups. Younger patients with moderate or severe BPH may wish to avoid surgery yet achieve symptom relief. Avoidance of troublesome side effects and the maintenance of libido and sexual function are also factors which will influence the choice of appropriate therapy.

\section{Dosage, administration, and formulations}

Dutasteride (Avodart ${ }^{\circledR}$ ) is indicated for the treatment of symptomatic $\mathrm{BPH}$ in men with an enlarged prostate to improve symptoms, reduce the risk of AUR, and reduce the risk of the need for BPH-related surgery. It is contraindicated for use in women and children and for patients with known hypersensitivity to dutasteride, other $5 \mathrm{ARI}$, or any component of the preparation. The recommended dose is one capsule $(0.5 \mathrm{mg}$ dutasteride) taken orally once a day and may be administered with or without food. 


\section{Clinical value}

There is now a range of options for the treatment of $\mathrm{BPH}$, including watchful waiting through to medical treatments, minimally invasive therapies, and surgery.

Men with mild BPH symptoms (as determined by AUA-SI) that are not bothersome may undergo watchful waiting in order to monitor any change in the condition. If there is progression to moderate or severe symptoms then any of the current treatment options may be considered. However, it is important for the physician and patient to discuss the various options together so that an appropriate treatment may be selected.

During clinical development, dutasteride has been shown to improve $\mathrm{BPH}$ symptoms and is also well tolerated. These findings have been confirmed during large open-label extension studies continuing for at least 2 years giving up to 4 years' clinical data with dutasteride. These observational studies have extended the findings that dutasteride reduces the risk of the most serious complications associated with $\mathrm{BPH}$, i.e. AUR and the need for surgery. This is achieved by dutasteride altering the natural history of BPH progression through significant reductions in prostate volume (achieved by rapid and near maximal suppression of circulating DHT levels).

Because dutasteride acts on the static component of bladder outlet obstruction, there is a delay in onset of symptom relief of up to 6 months. The combination of dutasteride and an alfa blocker (tamsulosin) has overcome this delay while limiting disease progression. Clearly this approach has advantages in the management of BPH. Up to 5 years follow-up data from the MTOPS study have shown that the combination of a 5ARI (finasteride) and alfa blocker was most effective in treating BPH (McConnell et al. 2003). Recent treatment guidelines have used these findings to recommend that combination therapy with 5ARls and alfa blockers are more beneficial than monotherapy (Roehrborn et al. 2003c; Madersbacher et al. 2004).

In conclusion, dutasteride has a place in therapy for those patients with a demonstrable prostate enlargement and the presence of LUTS. For rapid symptom relief, combination with an alfa blocker may be appropriate. There is strong evidence that dutasteride has a positive effect not only on disease-oriented outcomes (e.g. reductions in DHT levels and prostate volume), but also all important patient-oriented outcomes (including symptom relief, and avoidance of AUR and the need for surgery).

\section{References}

Andriole GL, Kirby R. Safety and tolerability of the dual $5 \alpha$-reductase inhibitor dutasteride in the treatment of benign prostatic hyperplasia. Eur Urol. 2003;44:82-88.

Andriole GL, Guess HA, Epstein JI, et al. Treatment with finasteride preserves usefulness of prostate-specific antigen in the detection of prostate cancer: results of a randomized, double-blind, placebo-controlled clinical trial. Urology. 1998;52:195-202.

Andriole GL, Roehrborn CG, Nickel C, et al. Effect of the dual $5 \alpha$-reductase inhibitor, dutasteride, on serum total PSA, free PSA and the ratio of F/T PSA. J Urol. 2002;167(no. 4 Suppl.):209-210.
Andriole GL, Ray P, Humphrey P, et al. The impact of dutasteride, a novel dual $5 a-r e d u c t a s e$ inhibitor, on both serum and intraprostatic androgens. Eur Urol Suppl. 2003;2:85

Barkin J, Guimarães M, Do Castelo V, et al. Dutasteride provides sustained symptom relief following short term combination treatment with tamsulosin. J Urol. 2002;167(no. 4 Suppl.):372.

Barkin J, Guimarães M, Jacobi G, et al. Alpha-blocker therapy can be withdrawn in the majority of men following initial combination therapy with the dual $5 \alpha$-reductase inhibitor dutasteride. Eur Urol. 2003;44:461-466.

Barry MJ, Williford WO, Chang Y, et al. Benign prostatic hyperplasia specific health measures in clinical research: how much change in the American Urological Association symptom index and the benign prostatic hyperplasia impact index is perceptible to patients? J Urol. 1995;154:1770-1774.

Boyle P, Roehrborn C, Andriole G, et al. The impact of dutasteride, a novel $5 \alpha-$ reductase inhibitor, on the hallmarks of $\mathrm{BPH}$. Progression and outcomes. Eur Urol Suppl. 2002a;1:107.

Boyle P, Siami P, Wachs BH, et al. Effect of dutasteride on the risk of acute urinary retention and the need for surgical treatment. J Urol. 2002b;167(no. 4 Suppl.):372.

Boyle P, Roehrborn CG, Marks LS, et al. Early use of dutasteride arrests prostate growth, improves clinical parameters and prevents complications in men with benign prostatic hyperplasia. J Urol. 2003a;169 (no. 4 Suppl.):477.

Boyle P, Roehrborn CG, Marks LS, et al. The novel dual $5 \alpha$-reductase inhibitor dutasteride is effective for the treatment and prevention of complications in men with a PV $30 \leq 40 \mathrm{cc}$ and $40 \mathrm{cc}$. Eur Urol Suppl. 2003b;2:160.

Bramson HN, Hermann D, Batchelor KW, et al. Unique preclinical characteristics of GG745, a potent dual inhibitor of 5AR. J Pharm Exp Ther. 1997;282:1496-1502.

Carson C, Harkaway R, Marks LS, et al. Effect of maximal dihydrotestosterone suppression with dutasteride on sexual function and gynecomastia. J Urol. 2003;169(no. 4 Suppl.):478.

Clark RV, Matsumoto AM. Bone density, bone metabolism markers and lipid profiles in healthy men are unaffected by the novel dual $5 \alpha$-reductase inhibitor dutasteride. J Urol. 2003;169(no. 4 Suppl.):479.

Clark R, Huffman C, Swerdloff R, et al. Potent DHT suppression by the novel dual $5 \alpha$-reductase inhibitor dutasteride does not affect bone density, bone metabolism or lipid profiles in healthy men. Eur Urol Suppl. 2003;2:160.

Clark RV, Hermann DJ, Cunningham GR, et al. Marked suppression of dihydrotestosterone in men with benign prostatic hyperplasia by dutasteride, a dual 5 $\alpha$-reductase inhibitor. J Clin Endocrinol Metab. 2004;89:2179-2184.

de la Rosette JJ, Kortmann BB, Rossi C, et al. Long-term risk of retreatment of patients using alpha-blockers for lower urinary tract symptoms. $J$ Urol. 2002;167:1734-1739.

Debruyne F, Barkin J, van Erps P, et al. Efficacy and safety of long-term treatment with the dual $5 \alpha$-reductase inhibitor dutasteride in men with symptomatic benign prostatic hyperplasia. Eur Urol. 2004;46:488-495.

Djavan B. Benign prostatic hyperplasia: where do we stand in the new millennium. Curr Opin Urol. 2002;12:1-2.

Dull P, Reagan RW, Bahnson RR. Managing benign prostatic hyperplasia. $\underline{A m}$ Fam Phys. 2002;66:77-84.

Emberton M. Earlier initiation of treatment with the dual $5 \alpha$ reductase inhibitor dutasteride reduces the risk of acute urinary retention and surgical intervention in men with benign prostatic hyperplasia. Eur Urol Suppl. 2004;3:88.

Gilling P, Jacobi G, Tammela T, et al. Efficacy of dutasteride and finasteride for the treatment of benign prostate hyperplasia: results of the 1-year Enlarged Prostate International Comparator Study (EPICS). BJU Int. 2005; 95(S1):1-38 (Abstract U051).

Hagerty J, Ginsberg P, Harkaway R. A prospective, comparative study of the onset of symptomatic benefit of dutasteride versus finasteride in men with benign prostatic hyperplasia in everyday clinical practice. Eur Urol Suppl. 2004;3:88.

Kaplan SA. AUA guidelines and their impact on the management of BPH: an update. Rev Urol. 2004;6(Suppl. 9):S46-S52. 
Madersbacher S, Alivizatos G, Nordling J, et al. EAU 2004 guidelines on assessment, therapy and follow-up of men with lower urinary tract symptoms suggestive of benign prostatic obstruction (BPH guidelines). Eur Urol. 2004;46:547-554.

McConnell JD, Bruskewitz R, Walsh P, et al. The effect of finasteride on the risk of acute urinary retention and the need for surgical treatment among men with benign prostatic hyperplasia. N Engl J Med. 1998;338:557-563.

McConnell JD, Roehrborn CG, Bautista OM, et al. The long-term effect of doxazosin, finasteride, and combination therapy on the clinical progression of benign prostatic hyperplasia. N Engl J Med. 2003;349:2387-2398.

O'Leary MP, Roehrborn C, Andriole G, et al. Improvements in benign prostatic hyperplasia-specific quality of life with dutasteride, the novel dual $5 \alpha$-reductase inhibitor. BJU Int. 2003;92:262-266.

Oesterling JE. Benign prostatic hyperplasia: medical and minimally invasive treatment options. N Engl J Med. 1995;332:99-109.

Pickard R, Emberton M, Neal DE. The management of men with acute urinary retention. Br J Urol. 1998;81:712-720.

Roehrborn CG. The epidemiology of acute urinary retention in benign prostatic hyperplasia. Rev Urol. 2001;3:187-192.

Roehrborn CG, Malice MP, Cook TJ, et al. Clinical predictors of spontaneous acute urinary retention in men with LUTS and clinical BPH: a comprehensive analysis of the pooled placebo groups of several large clinical trials. Urology. 2001;58:210-216.

Roehrborn CG, Andriole G, Boyle P, et al. Effect of the dual 5 alpha-reductase inhibitor dutasteride on endocrine parameters and prostate volume. Eur Urol Suppl. 2002a;1:107.

Roehrborn CG, Andriole GL, Nickel C, et al. Effect of the dual $5 \alpha$-reductase inhibitor dutasteride on endocrine parameters. J Urol. 2002b;167(no. 4 Suppl.) (Abstract 104013).

Roehrborn CG, Boyle P, Nickel JC, et al. Efficacy and safety of a dual inhibitor of 5-alpha-reductase types 1 and 2 (dutasteride) in men with benign prostatic hyperplasia. Urology. 2002c;60:434-441.
Roehrborn CG, Ramsdell J, Siami P, et al. Prostate volume at baseline predicts the margin of therapeutic response with the $5 \alpha$-reductase inhibitor, dutasteride. J Urol. 2002d;167(no. 4 Suppl.) 373.

Roehrborn C, Andriole G, Schalken J, et al. Dutasteride, a novel dual $5 \alpha-$ reductase inhibitor, reduces serum DHT to a greater extent versus finasteride and achieves finasteride maximal reduction in a larger proportion of patients. Eur Urol Suppl. 2003a;2:161.

Roehrborn CG, Boyle P, Nickel C. Dutasteride provides sustained and continued improvement in BPH-related symptoms over 4 years. J Urol. 2003b;169(no. 4 Suppl.):333.

Roehrborn CG, McConnell JD, Barry MJ, et al. Guideline on the management of benign prostatic hyperplasia $(B P H)$. American Urological Association Education and Research, Inc. 2003c. Available at:

http://www.auanet.org/guidelines/bph.cfm.

Roehrborn CG, de la Rosette J, Andriole GL. The utility of serial PSA measurements for the diagnosis of prostate cancer is preserved in men treated with the dual 5-alpha reductase inhibitor dutasteride. J Urol. 2004a;171(no. 4 Suppl.) (Abstract 2095).

Roehrborn CG, Marks LS, Fenter T, et al. Efficacy and safety of dutasteride in the four-year treatment of men with benign prostatic hyperplasia. Urology. 2004b;63:709-715.

Tammela T, Barkin J, Roehrborn CG, et al. Long-term dutasteride therapy results in sustained reductions in total prostate volume in men with symptomatic benign prostatic hyperplasia. Eur Urol Suppl. 2004;3:88.

Watson V, Ryan M, Brown CT, et al. Eliciting preferences for drug treatment of lower urinary tract symptoms associated with benign prostatic hyperplasia. J Urol. 2004;172:2321-2325.

Zlotta AR, Djavan B. Minimally invasive therapies for benign prostatic hyperplasia in the new millennium: long-term data. Curr Opin Urol. 2002;12:7-14.

Correspondence: Andrew Thomson, Core Medical Publishing, Mere House, Brook Street, Knutsford, Cheshire WA16 8GP, UK or at editor@coreevidence.com 